ARTICLE

Received 19 Dec 2013 | Accepted 22 May 2014 | Published 23 Jun 2014

DOI: $10.1038 /$ ncomms5201

OPEN

\title{
AmiA is a penicillin target enzyme with dual activity in the intracellular pathogen Chlamydia pneumoniae
}

Anna Klöckner ${ }^{1, \star}$, Christian Otten ${ }^{1, \star}$, Adeline Derouaux ${ }^{2, \dagger}$, Waldemar Vollmer ${ }^{2}$, Henrike Bühl ${ }^{1}$, Stefania De Benedetti ${ }^{1}$, Daniela Münch ${ }^{1}$, Michaele Josten ${ }^{1}$, Katja Mölleken ${ }^{3}$, Hans-Georg Sahl ${ }^{1}$ $\&$ Beate Henrichfreise ${ }^{1}$

Intracellular Chlamydiaceae do not need to resist osmotic challenges and a functional cell wall was not detected in these pathogens. Nevertheless, a recent study revealed evidence for circular peptidoglycan-like structures in Chlamydiaceae and penicillin inhibits cytokinesis, a phenomenon known as the chlamydial anomaly. Here, by characterizing a cell wall precursorprocessing enzyme, we provide insights into the mechanisms underlying this mystery. We show that AmiA from Chlamydia pneumoniae separates daughter cells in an Escherichia coli amidase mutant. Contrary to homologues from free-living bacteria, chlamydial AmiA uses lipid II as a substrate and has dual activity, acting as an amidase and a carboxypeptidase. The latter function is penicillin sensitive and assigned to a penicillin-binding protein motif. Consistent with the lack of a regulatory domain in AmiA, chlamydial CPn0902, annotated as $\mathrm{NIpD}$, is a carboxypeptidase, rather than an amidase activator, which is the case for $E$. coli NIpD. Functional conservation of AmiA implicates a role in cytokinesis and host response modulation.

\footnotetext{
${ }^{1}$ Institute for Medical Microbiology, Immunology and Parasitology (IMMIP), Pharmaceutical Microbiology, University of Bonn, 53115 Bonn, Germany. ${ }^{2}$ The Centre for Bacterial Cell Biology, Institute for Cell and Molecular Biosciences, Newcastle University, Newcastle upon Tyne, NE2 4AX, UK. ${ }^{3}$ Institute of Functional Microbial Genomics, University of Düsseldorf, 40225 Düsseldorf, Germany. ${ }^{\star}$ These authors contributed equally to this work. $\dagger$ Present address: GIGA-Research, Molecular Biology and Genetic Engineering Unit, University of Liège, B-4000 Liège, Belgium. Correspondence and requests for materials should be addressed to B.H. (email: bhenrich@uni-bonn.de).
} 
C hlamydiaceae are characterized by an obligate intracellular lifestyle and a unique biphasic developmental cycle. Despite the economic and public health importance of ocular, respiratory and sexually transmitted diseases caused by Chlamydiaceae, our knowledge about their biology at a molecular level is still limited. One crucial aspect of chlamydial biology that remains to be elucidated is the long known, yet still enigmatic chlamydial anomaly: for endobacteria such as Chlamydiaceae, there is no need to maintain osmotic stabilization by means of a cell wall, and a functional cell wall has not been detected in these pathogens ${ }^{1}$. Nevertheless, antibiotics that target cell wall biosynthesis are active against Chlamydiaceae ${ }^{1}$. Penicillin has no bactericidal effect on Chlamydiaceae but induces a reversible state of persistence, a phenomenon that is observed during chronic, recurring infections with Chlamydia trachomatis and C. pneumoniae ${ }^{2}$.

The bacterial mesh-like cell wall consists of peptidoglycan (PG), a polymer of sugar chains that are cross-linked via flexible peptide bridges. PG is evolutionary conserved in eubacteria with the exception of some obligate intracellular species. Biosynthesis of PG takes place in three compartments of bacterial cells ${ }^{3}$. In the cytoplasm, formation of the soluble precursors UDP-Nacetylglucosamine and UDP- $N$-acetylmuramyl-pentapeptide is catalysed. At the inner leaflet of the cytoplasmic membrane, the two precursors are assembled with the lipid carrier bactoprenolphosphate (undecaprenyl-P) to form lipid II, the completed cell wall-building block. Lipid II is then translocated to the outside of the cell and incorporated into the PG network releasing undecaprenyl-PP.

Prokaryotic cell division is driven by $>10$ essential proteins, which assemble at the septum to form the divisome ${ }^{3-5}$. Briefly, the following stages of cell division are recognized in $E$. coli: the tubulin orthologue Fts $\mathrm{Z}$ forms a ring-like structure ( $\mathrm{Z}$ ring) at midcell and initiates divisome assembly by attracting a set of 'early' proteins to form a cytoplasmic apparatus linked to the membrane; then enzymes for lipid II biosynthesis and presumably for PG biosynthesis localize to the midcell to carry out 'preseptal' elongation, a process in which the actin orthologue MreB might participate; additional elements co-ordinate chromosome segregation and build an extracellular connector for outer membrane constriction; finally, proteins involved in the incorporation (for example, transpeptidase FtsI (penicillinbinding protein (PBP) 3) and flippase FtsW) and remodelling (amidases) of septal PG assemble. The process ends with cell segregation.

The three periplasmic $N$-acetylmuramyl-L-alanine amidases AmiA, AmiB and AmiC have been shown to be important for cell separation in E. coli. Double- and triple-amidase mutants form cell chains to varying extents ${ }^{6,7}$. The chain-forming amidase mutants constrict the inner membrane, fail to split septal PG, are connected by shared PG layers and partially constrict their outer membranes ${ }^{6,7}$. In contrast to $\mathrm{AmiB}$ and $\mathrm{AmiC}, \mathrm{AmiA}$ is not localized to the septum ${ }^{3}$. Nevertheless, E. coli double mutants with only AmiA are still able to partially separate ${ }^{6}$. AmiA, AmiB and AmiC are members of the zinc-dependent $N$-acetylmuramylL-Ala amidase 3 family and catalyse the release of peptide moieties from PG by cleaving the amide bond between the lactyl group of muramic acid and the amino group of L-alanine. In E. coli the amidases AmiA, AmiB and AmiC need to be activated by LytM domain proteins to efficiently hydrolyse septal PG. EnvC has been shown to serve as a specific activator for AmiA and $\mathrm{AmiB}$, and $\mathrm{NlpD}$ specifically activates $\mathrm{AmiC}^{8}$. In previous studies, E. coli AmiA has been shown to hydrolyse polymeric $\mathrm{PG}^{8}$, as well as glycan strands of varying chain length consisting of at least two transglycosylated lipid II cell wall-building blocks in a zincdependent fashion in vitro. Monomeric lipid II was not degraded by $E$. coli AmiA, suggesting that the enzyme requires at least a tetrasaccharide as a substrate?

Despite massive reduction in the genome size, a nearly complete pathway for lipid II biosynthesis has been found in Chlamydiaceae genomes ${ }^{1}$, and activity of enzymes catalysing cytoplasmic steps (MurA, MurC/Ddl, CT390, DapF, MurE, GlyA and MurF) and the two membrane-linked steps (MraY and MurG) of cell wall precursor synthesis has been demonstrated ${ }^{1,10-14}$. Chlamydiaceae genomes retained two periplasmic enzymes that are involved in side-wall (PBP2) and septal (FtsI (PBP3)) incorporation of lipid II building blocks in free-living rod-shaped bacteria such as E. coli. Moreover, the human pathogens harbour a rudimentary set of divisome proteins, lacking the almost ubiquitous central organizer FtsZ, but including FtsW, FtsI (PBP3) and FtsK, and possess, despite their spherical shape, the cytoskeletal protein $\mathrm{MreB}^{1,15}$. AmiA is the only amidase encoded by Chlamydiaceae genomes and cell wall remodelling enzymes such as endopeptidases and lytic transglycosylases have not been identified ${ }^{1}$.

We discussed that maintaining biosynthesis of the cell wallbuilding block lipid II in cell wall-lacking 'minimal bacteria' such as Chlamydiaceae may reflect a vital role of the lipid II pathway in prokaryotic cell division ${ }^{13}$. Moreover, we proposed that MreB is involved in orchestration of lipid II biosynthesis at the septum and as such may be necessary for maintaining a functional divisome machinery in Chlamydiaceae ${ }^{15}$. Recently, a cell wall sacculus was detected in the evolutionary earlier, genomically less reduced environmental chlamydiae Protochlamydia ${ }^{16}$. However, consistent with our model described above, MreB and its interacting partner RodZ from the environmental chlamydial genus Waddlia have been shown to localize to the septum ${ }^{17}$, and in Chlamydiaceae no cell wall but a distinct circularly shaped structure was found. This structure was suggested to consist of rudimentary PG-like material that is localized to the septum of dividing cells ${ }^{18}$.

The aim of this study was to gain first mechanistic insights in processing of chlamydial lipid II at the division site. Here, we demonstrate that amidase AmiA is functionally conserved in Chlamydiaceae and capable of using the monomeric cell wallbuilding block lipid II as a substrate for amidase and novel penicillin-sensitive DD-carboxypeptidase (DD-CPase) activity in vitro. AmiA-driven processing of lipid II might be crucial for a complete lipid II biosynthesis and recycling cycle, and thus for maintaining coordinated cell division in Chlamydiaceae. Moreover, lipid II processing might modulate host response to muropeptides.

\section{Results}

AmiA from C. pneumoniae is functional in E. coli. AmiA from C. pneumoniae (AmiACp) and E. coli show 32\% amino-acid sequence identity. If AmiACp is capable of degrading PG, as shown before for the homologue of $E$. coli ${ }^{8}$, the enzyme might lyse bacterial cells. Overexpression of AmiACP in the periplasm of E. coli was achieved by fusion to the OmpA leader peptide and resulted in lysis of the producer strain after induction (Fig. 1a). To investigate functionality of AmiACp in cell separation of freeliving bacteria, we performed complementation assays using an E. coli $\triangle a m i A B C$ triple-knockout mutant harbouring an arabinose-inducible amiC gene on a plasmid (Fig. 1b). When grown in the presence of glucose, the cells fail to separate during cell division and form long chains (Fig. 1c). The expression of AmiACP resulted in separated rod-shaped cells (Fig. 1c). Quantitative analysis of the experiments revealed that virtually all cells of the amidase triple mutant were present in chains (Fig. 1d). Upon induction of AmiACp expression, the separation 


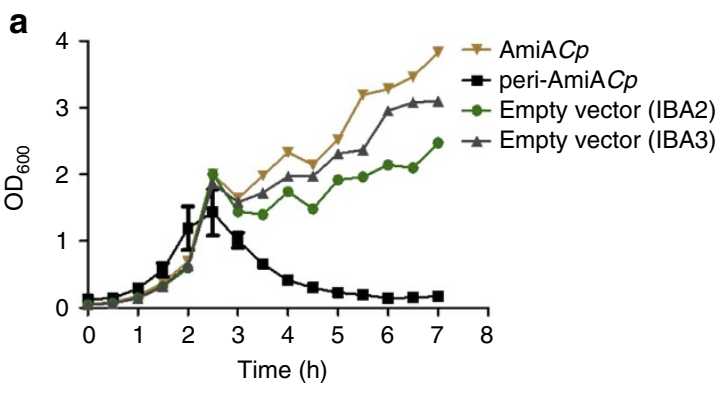

C
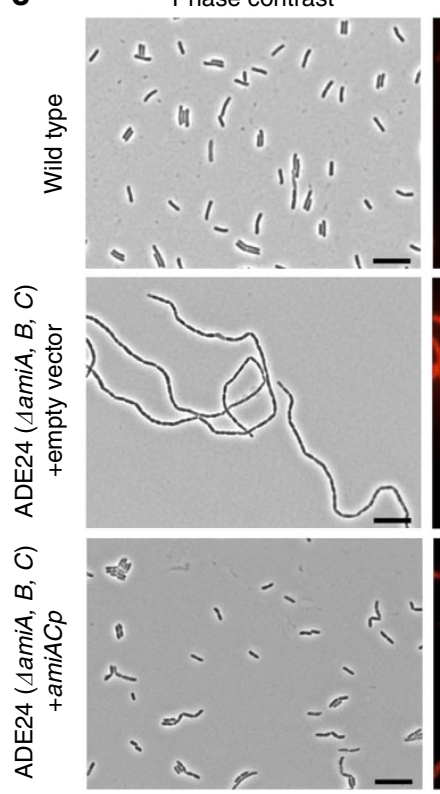

Nile red

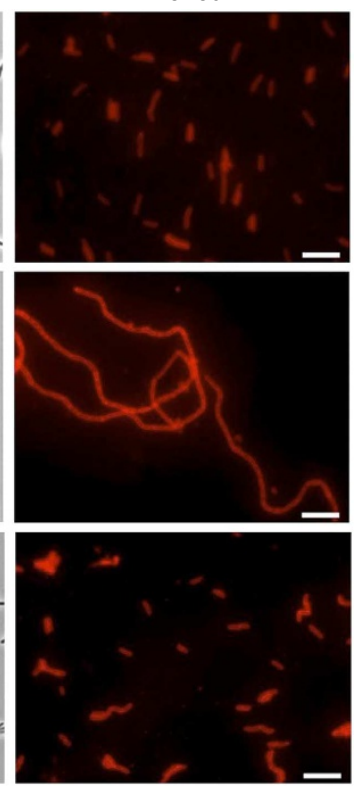

b

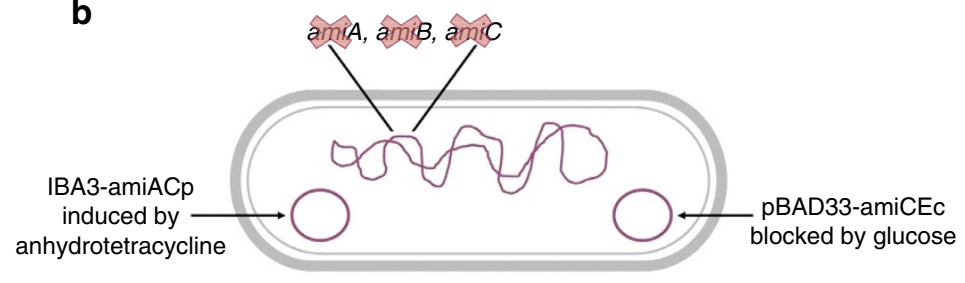

d
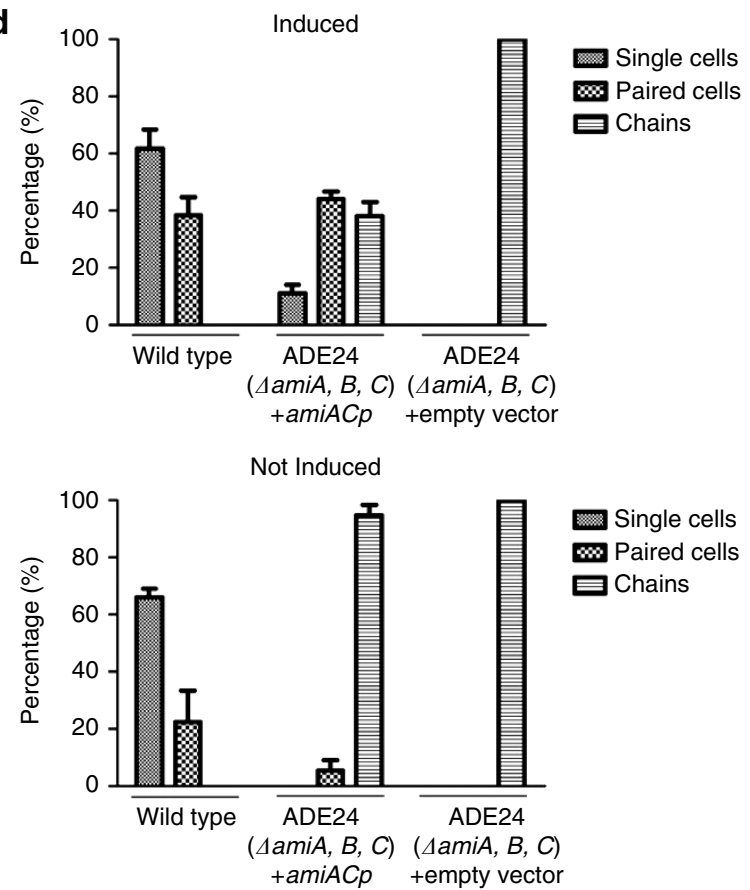

Figure 1 | Activity of AmiACp in E. coli. Growth kinetics of $E$. col JM83 containing chlamydial amiA constructs. (a) AmiA expression was induced at an $\mathrm{OD}_{600}$ of 0.6 . Periplasmic expression of AmiACp (peri-AmiACp) resulted in lysis of $E$. coli JM83. Without being fused to the OmpA leader peptide $($ Ami $A C p), A m i A$ did not accumulate sufficiently at the periplasm to cause lysis of the expression strain. Error bars indicate $\pm s . d$. ( $n=3$ ). Complementation of E. coli $\triangle a m i A B C$ triple-knockout mutant ADE24 by chlamydial AmiA (b-d). Illustration of E. coli ADE24 (b). The mutant was transformed with plasmid IBA3-amiACp that allows for AHT-induced expression of AmiACp; microscopy (c) and quantative (d) analyses of the experiments: in the presence of glucose and $\mathrm{AHT}$, expression of AmiC from $E$. coli was blocked and complementation of the chain-forming triple-amidase mutant by AmiACp resulted in separated rod-shaped cells. Error bars are mean \pm s.d. $(n=3)$. Scale bar, $10 \mu \mathrm{m}$. A control experiment with AmiA from E. coli is presented in Supplementary Fig. 5.

of daughter cells was rescued and $55 \%$ of the cells were single or paired (Fig. 1d). These data are in agreement with 30-40\% chains observed in an E. coli $\triangle a m i B \triangle a m i C$ mutant containing $\mathrm{AmiA}^{6}$. In total, these findings indicate that AmiACp can perform daughter cell separation in $E$. coli, presumably by the degradation of septal PG during cell division.

AmiACp shows amidase activity on the PG and lipid II precursor. Purified AmiACp was tested for degradation of PG using a remazol dye-release assay. As shown before for $E$. coli amidases ${ }^{8}$, AmiACp released remazol-stained reaction products into the supernatant from the PG sacculi (Fig. 2a,b). A control protein (DD-CPase VanY) that removes D-Ala from the peptide side chains, did not release the dye.

Chlamydiaceae do not contain detectable quantities of PG but retained the biosynthesis pathway of the cell wall-building block lipid $\mathrm{II}^{13}$. We tested whether chlamydial AmiA can remove the pentapeptide side chain from the membrane-bound cell wall precursor. Thin-layer chromatography (TLC) analysis of lipid II reaction products combined with mass spectrometry (MS) revealed that AmiACP was capable of using lipid II as a substrate for amidase activity and cleaved the peptide side chain from the sugar moieties of lipid II (Fig. 2c-f).

In summary, AmiACp is an $N$-acetylmuramyl-L-alanine amidase using polymeric PG and monomeric lipid II as a substrate in vitro.

AmiACp has an additional function as DD-CPase. Surprisingly, our TLC/MS analysis showed that chlamydial AmiA exhibited dual enzymatic activity on lipid II in vitro. In addition to the amidase product, we identified lipid II with a tetrapeptide, indicating that cleavage of the terminal D-Ala residues had occurred (Fig. 2c-f). Therefore, AmiA has also DD-CPase activity. It was possible that the heterologous AmiA produced in E. coli was contaminated by host DD-CPases (class C PBPs). To test this possibility, we performed several control experiments. A mock protein purification from the E. coli strain harbouring the empty vector had no detectable DD-CPase activity (Fig. 2c) and we did not detect any PBPs in the AmiACp preparation when labelling with fluorescent penicillin bocillin FL (Supplementary Fig. 1), making it unlikely that the DD-CPase detected was due to contaminating host PBPs. The enzyme had no beta-lactamase activity 
a

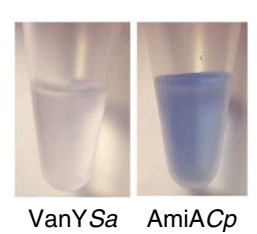

b 0.8

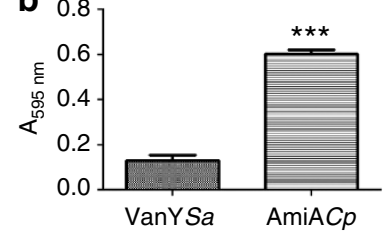

d

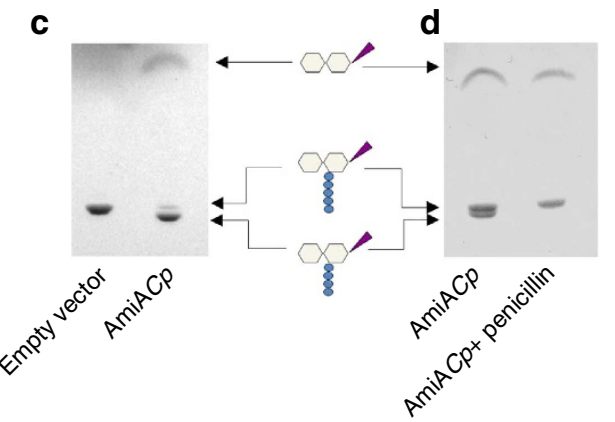

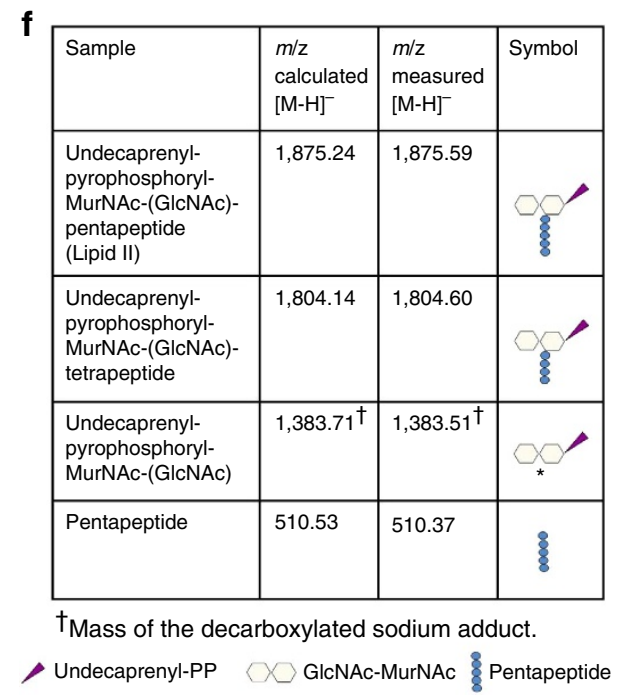

e Empty vector
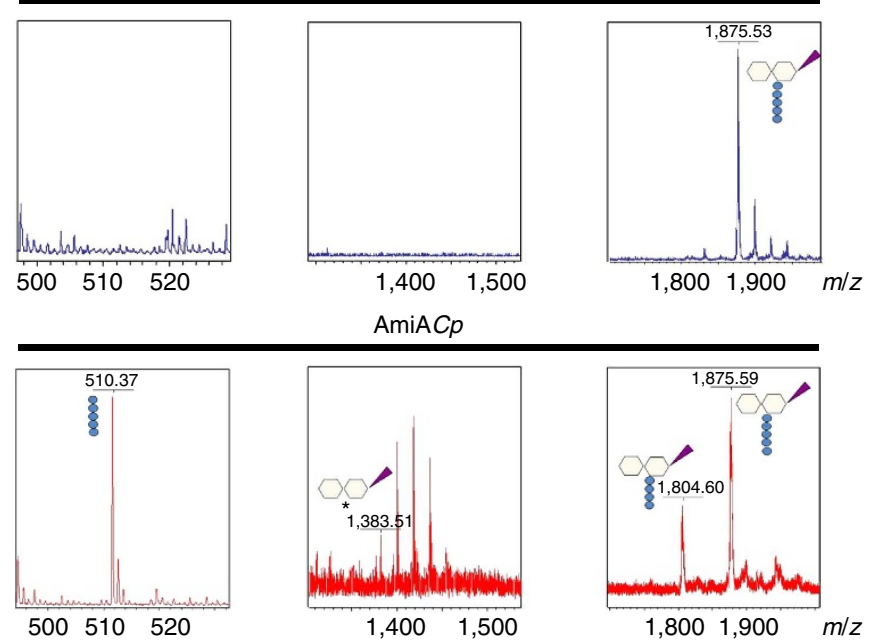

Figure 2 | In vitro activity of AmiACp. The enzyme used PG as well as monomeric lipid II as a substrate. Dye-release assay on the degradation of PG: released reaction products after incubation of remazol-stained PG with control enzyme VanY from Staphylococcus aureus (VanYSa) and AmiACp (a) and photometric analysis of supernatants $(\mathbf{b})$. Error bars indicate \pm s.d. $(n=3)$. Unpaired $t$-test revealed statistical significance in comparision with VanY control, two-tailed ${ }^{\star \star \star} P$-value $\leq 0.0001$. TLC analysis $(\mathbf{c}, \mathbf{d})$ and MS $(\mathbf{e}, \mathbf{f})$ of lipid II reaction products. AmiACp showed novel amidase and DD-CPase activity on lipid II. The latter function was sensitive to penicillin. ${ }^{\star}$ The $0-1$-carboxyethyl-residue at position 3 in the MurNAc moiety of the amidase reaction product undecaprenyl-pyrophosphoryl-MurNAc-GlcNAc is decarboxylated, as usually observed with the matrix 6-Aza-2-thiothymine ${ }^{46}$. Additional peaks in the spectrum for the decarboxylated undecaprenyl-pyrophosphoryl-MurNAc-GIcNAc sodium adduct are attributed to the addition of water to conjugated double bonds in the undecaprenyl moiety (mass shift of $18 \mathrm{Da}$ ). (GlcNAc: N-acetylglucosamine; MurNAc: N-acetylmuramic acid).

in a nitrocefin hydrolysis-based test system (Supplementary Fig. 2), indicating that the detection of possibly contaminating PBPs was not impaired by hydrolysis of bocillin FL. Of note, DD-CPase activity of AmiACp was sensitive to penicillin and blocked in a molar ratio of 1:1 (protein:inhibitor), whereas amidase activity was not impaired in the presence of the betalactam (Fig. 2d; Supplementary Fig. 3). Our results identify AmiACp as a novel DD-CPase and, besides PBP2, PBP3 and PBP6, as an additional so far unknown target of beta-lactams in Chlamydiaceae.

Inactivation of the amidase active site. The active site of $E$. coli $\mathrm{AmiA}$ is comprised by three zinc coordinating residues (H65, E80 and H133) and E242 that is predicted to serve as a general base catalyst ${ }^{9}$. We found all residues to be conserved in AmiACP (Fig. 3) and replaced H67, H136 and E207 (corresponding to H65, H133 and E242 in E. coli) with alanine to explore their functional role in amidase activity. Expression of AmiAH67ACP and AmiAH136ACp mutants at the periplasm impaired growth of the E. coli expression strain but did not lead to cell lysis (Fig. 4a). The exchange of E207 had no effect on the lytic activity of AmiACp. These results indicate that both histidine residues are essential for amidase activity of AmiACp, as shown before for the corresponding residues in E. coli ${ }^{9}$. In contrast, the glutamic acid, which is necessary for AmiA activity in $E$. coli ${ }^{9}$, is not needed for amidase function in the chlamydial homologue. We further investigated whether inactivation of the amidase active site affects DD-CPase activity. The AmiAH67ACp mutant was still capable of cleaving terminal D-Ala residues from the pentapetide side chain of lipid II, implicating that both activities are independent from each other (Supplementary Fig. 4a).

Identification of the DD-CPase active site. Penicillin-sensitive PBP DD-CPases, including VanY $Y_{D}$ DD-CPase from vancomycin- 


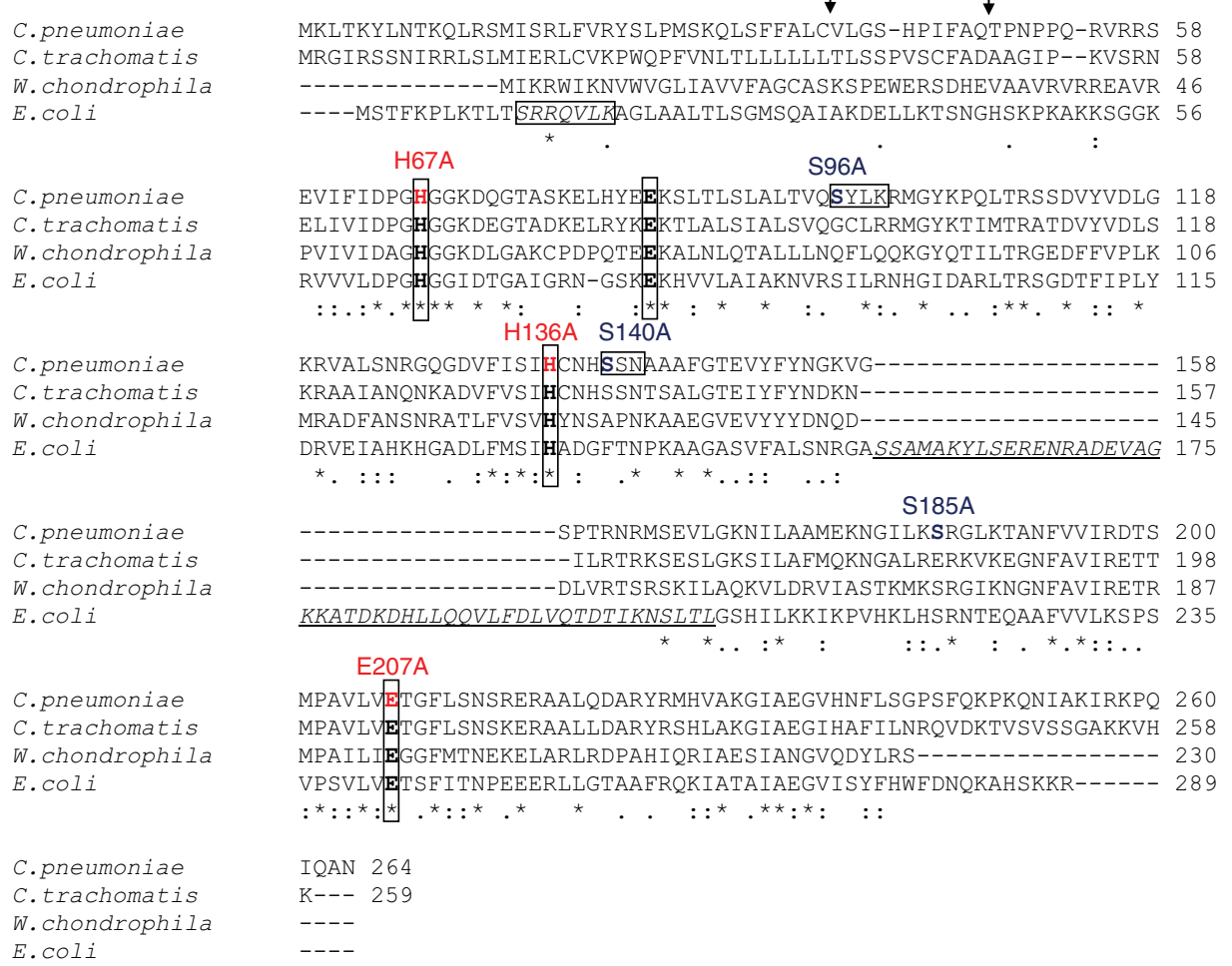

Figure 3 | Sequence alignment of AmiA from E. coli and selected Chlamydiales species. The amidase active site of E. coli AmiA contains three zinccoordinating residues ( $\mathrm{H} 65, \mathrm{E} 80$ and $\mathrm{H} 133$ ), and $\mathrm{E} 242$ that is predicted to serve as a general base catalyst ${ }^{9}$. These active site residues (in bold and boxed) are highly conserved in the Chlamydiales species. Of note, the Chlamydiales AmiA proteins lack a domain with predicted autoregulatory functions (underlined and in italics) that is exclusively found in cell division amidases AmiA, AmiB and AmiC and contains an $\alpha$-helix (S157-V173 in E. coli AmiA, corresponding to D280-L296 in AmiB from B. henselae) occluding the active site. During cell division, the regulatory domain is proposed to interact with LytM domain factors to relieve autoinhibition by a conformational switch ${ }^{25}$. PBP DD-CPases have three typical motifs, $S \times x K, S(Y) x N$ and $K(H, R) T(S) G$, that are essential for substrate recognition and catalysis. AmiA from C. pneumoniae contains an SxxK and an SxN motif but lacks the KTG motif. The signal peptide from E. coli contains an SRRxFLK (with $\mathrm{x}$ being a polar amino acid) consensus motif (boxed and in italics) that directs AmiA to the Tat translocation system. Consistent with the absence of a Tat system in chlamydiae, AmiA from C. pneumoniae does not contain a functional Tat motif. The solid arrow and the dashed arrow point to the signal peptidase cleavage sites in AmiA from C. pneumoniae revealed by MS analysis and predicted by SignalP 32 , respectively.
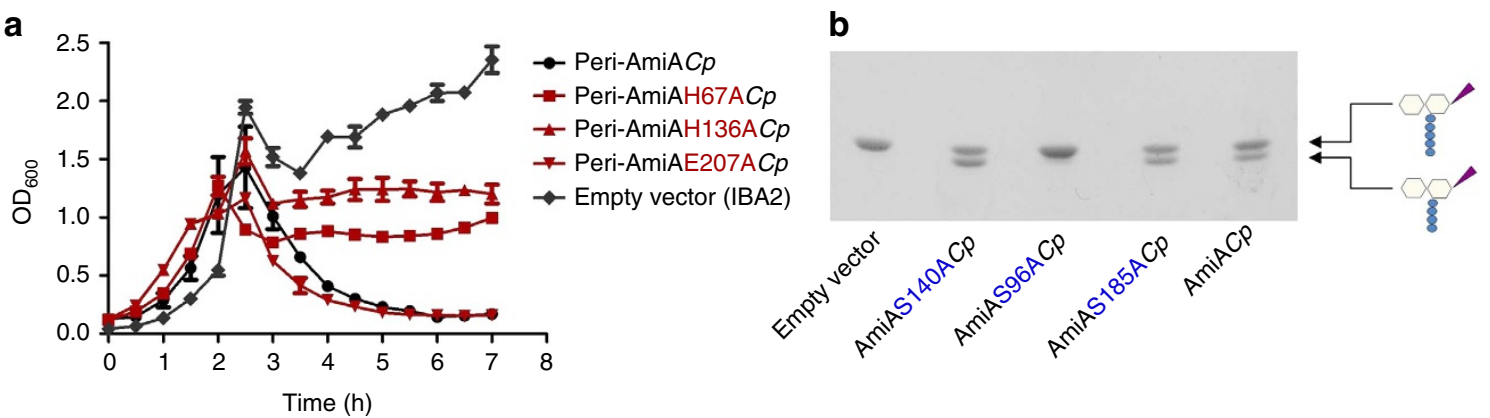

Figure 4 | Characterization of active sites. Studies of the amidase active site (a). The zinc-coordinating His residues are essential for the lytic activity of AmiACp. Error bars indicate \pm s.d. $(n=3)$. Discovery of the amino acids involved in DD-CPase activity (b). The exchange of residue S96 in the SxxK tetrad leads to a loss of function.

resistant Enterococcus faecium ${ }^{19}$, are acyl-serine transferases and contain three typical motifs, SxxK, $\mathrm{S}(\mathrm{Y}) \mathrm{xN}$ and $\mathrm{K}(\mathrm{H}, \mathrm{R}) \mathrm{T}(\mathrm{S}) \mathrm{G}$, that are essential for substrate recognition and catalysis ${ }^{20}$. AmiACp has an SxxK (S96-Y97-L98-K99) and an SxN motif (S140-S141-N142), but no KTG motif (Fig. 3). The zincdependent DD-CPase of Streptomyces albus as well as PdcA from Myxococcus xanthus ${ }^{20}$ and $\mathrm{VanY}^{21}$ and $\mathrm{VanY}_{\mathrm{B}}{ }^{22}$ from enterococci are DD-CPases that are beta-lactam insensitive.
AmiACp has neither the zinc-binding motif $\mathrm{H}(\mathrm{x} 42 \mathrm{aa}) \mathrm{HxH}$ of the S. albus enzyme ${ }^{23}$ nor the motifs $\operatorname{SxHxxGxA(S)\times D}$ and $\mathrm{EP}(\mathrm{W}) \mathrm{WH}$ conserved in the DD-CPases $\operatorname{Van} Y$, Van YB and $P d c A^{20,24}$. Recently, the first structure of a cell separation amidase, AmiB from Bartonella henselae, was solved ${ }^{25}$. We conducted a search for similar structures to $\mathrm{AmiB}$ from $B$. henselae using the DALI server ${ }^{26}$ and found matches with members of the carboxypeptidase A family ( $Z$-scores ranging 
from 9.8-8). This zinc metalloprotease family is dominated by mammalian enzymes but comprises a few proteins of bacterial origin such as the $\gamma$-D-glutamyl-(L)-mDAP-hydrolysing peptidase I from Bacillus sphaericus ${ }^{27}$. To identify amino acids that are involved in the DD-CPase function of AmiACp, we replaced the serine residues S96 and S140 in the two PBP homologous motifs SxxK and SxN as well as the residue S185, which is conserved in AmiA from E. coli and Chlamydiaceae and found in peptidase I from B. sphaericus. The replacement of S96 abolished DD-CPase activity, whereas the other mutations had no effect (Fig. 4b). These results provide further proof that the observed activity was not owing to a contaminating host $\mathrm{PBP}$ in the purified protein. The penicillin-sensitive DD-CPase function seems to be conferred solely by an SxxK motif independently from the apparently not essential SxN triad and the absent KTG motif. This is different from conventional bacterial PBPs whose activity depend, in addition to the SxxK tetrad, on a functional SxN motif and which typically contain a KTG motif as well ${ }^{28}$. The DD-CPase active site mutant AmiAS96ACp was not impaired in amidase activity, providing further evidence that both enzymatic activites of AmiACP are independent from each other (Supplementary Fig. 4b).

Secretion of AmiACp. AmiA from E. coli contains a twinarginine translocation (Tat) motif and is transported to the periplasm by the Tat system ${ }^{29}$. Consistent with the absence of a
Tat system in Chlamydiaceae, AmiACp does not have an

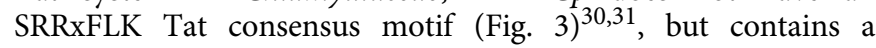
putative signal peptide. Without being fused to the OmpA leader peptide, the overexpressed AmiACp did not accumulate efficiently in the periplasm to lyse the $E$. coli producer strain (Fig. 1a, AmiACp). Nevertheless, in the complementation assay, native AmiACp was able to rescue cell separation in the $E$. coli amidase triple mutant (Fig. 1c,d), whereas AmiA $\triangle S P C p$, which lacks the putative signal peptide, failed to complement the mutant (Supplementary Fig. 5). MS of the heterologously expressed native AmiACp revealed cleavage of the signal peptide in E. coli between residues 37 and 38 and not further downstream as frequently found for Tat signal peptides and predicted by SignalP (Fig. 3) $)^{32}$. Thus, our data indicate that AmiACp contains a functional Sec signal peptide.

Chlamydial AmiA lacks an autoregulatory domain. The active sites of the cell division amidases AmiA, AmiB and AmiC from Gram-negative bacteria are likely to be occluded by a conserved $\alpha$-helix, as previously shown for AmiB from $B$. henselae ${ }^{25}$. These amidases have low basal in vitro activity and need to be activated by EnvC or NlpD. The LytM-containing septal ring factors are thought to specifically interact with the autoregulatory domain of the corresponding enzymes (EnvC with AmiA and AmiB and $\mathrm{NlpD}$ with $\mathrm{AmiC}$ ) to promote the release of the regulatory helix from the active $\operatorname{site}^{25}$. Sequence analysis showed that AmiACP

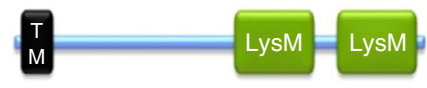

b

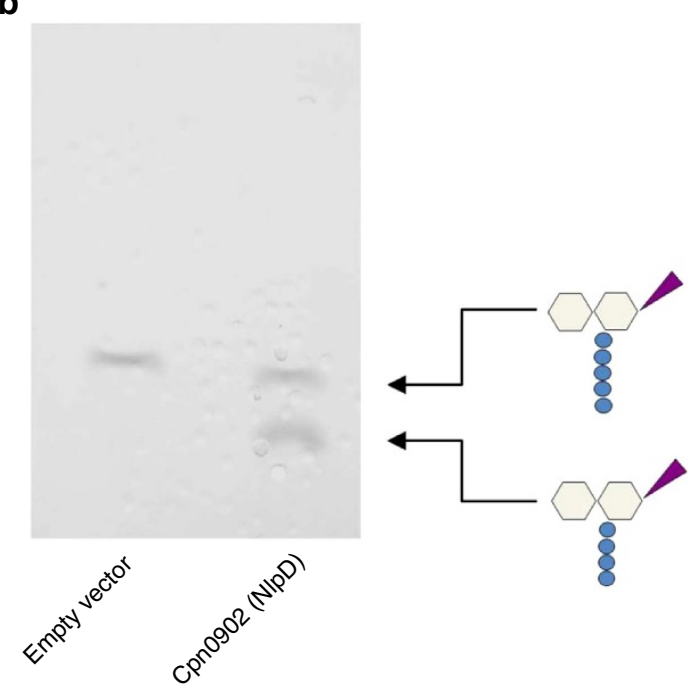

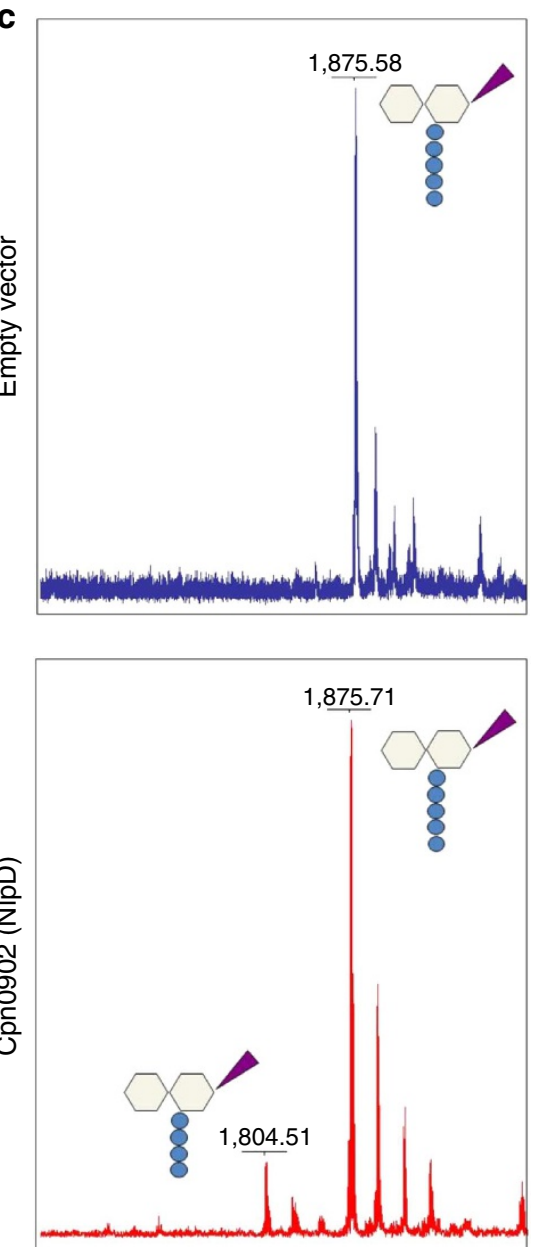

Figure 5 | Structure and in vitro activity of Cpn0902 (NIpD). Conserved LysM (peptidoglycan-binding lysin subgroup) domains (identified using InterPro protein sequence analysis and classification tool, EMBL-EBI ${ }^{47}$ ) and a transmembrane domain (TM) are depicted (predicted by TMHMM software, Technical University of Denmark ${ }^{48}$ ) (a). TLC (b) and MS (c) analysis of reaction products revealed DD-CPase activity of Cpn0902 on lipid II. 


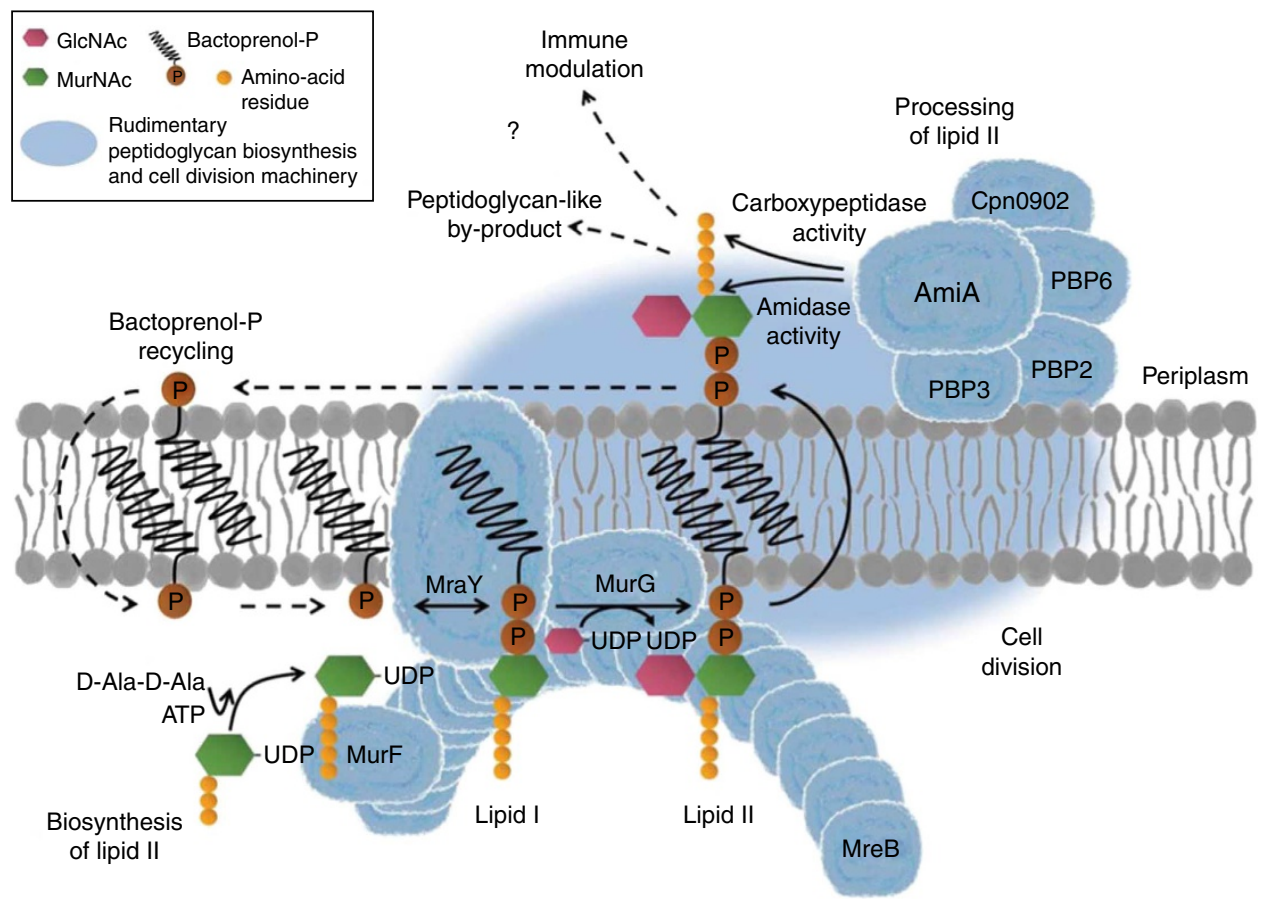

Figure 6 | Proposed model for the role of the conserved lipid II pathway in maintaining a functional cell division machinery and contributing to modulation of host response in Chlamydiaceae. A complete cycle of lipid II biosynthesis, processing and recycling needs to be maintained for coordinated function of the cell division machinery. Structural protein MreB (in interaction with RodZ) functionally organizes MurF, MraY and MurG, three key components in lipid II biosynthesis, at the septum. The synthesized precursor is translocated to the outside and processed by a rudimentary cell wall biosynthesis/cell division machinery. DD-CPase activity of AmiA, together with PBP6 and Cpn0902 (NIpD), might orchestrate DD-CPase functions in chlamydial cell division. Amidase activity of AmiA would play a central role in processing lipid II by releasing the pentapeptide side chain to allow for (i) transpeptidation of pentapeptides catalysed by monofunctional transpeptidases Ftsl and PBP2 and for (ii) regulation of host immune response due to blocking of Nod2 sensing. FtsZ as well as transglycosylases, endopeptidases and pyrophosphorylases are not found in Chlamydiaceae. Dashed arrows and question marks indicate steps of the proposed pathway that remain to be elucidated. (GlcNAc: $\mathrm{N}$-acetylglucosamine; MurNAc: $\mathrm{N}$-acetylmuramic acid).

lacks this autoinhibitory domain ( $\alpha$-helix 5, aa 292-340 in E. coli AmiB, see alignment Fig. 3) and is unlikely to be autoregulated comparable to phage and mother cell lysis amidases such as CD27L, PLY-PSA and CwlVc ${ }^{25}$. The absence of an autoregulatory helix in AmiACP is consistent with the fact that Chlamydiaceae harbour neither the corresponding activator EnvC nor the AmiC activator NlpD. Moreover, the rudimentary divisome machinery of Chlamydiaceae lacks FtsE and FtsX, which have been shown to recruit the AmiA and AmiB activator EnvC to the septum in E. coli ${ }^{33}$. CPn0902, annotated as NlpD in C. pneumoniae, does not contain a LytM domain but two putative PG-binding LysM domains in the C-terminus. The companion paper by Frandi et al. ${ }^{34}$ demonstrates that expression of Waddlia NlpD in E. coli increases the ratio of muro-tetrapeptide monomers to dimers in the PG. Consistently, purified Cpn0902 (NlpD) from C. pneumoniae was capable of releasing terminal D-Ala residues from the pentapeptide side chain of lipid II showing that the protein is rather a DD-CPase than an amidase-activating factor (Fig. 5).

\section{Discussion}

Since the first description of the chlamydial anomaly in 1993, several studies proved the functionality of early cytoplasmic steps ${ }^{1,10-12,14}$ and of the two cytoplasm membrane-associated steps $^{13}$ of lipid II biosynthesis in Chlamydiaceae. This is the first description of a chlamydial lipid II-processing enzyme in the periplasm.

In reticulate bodies, the replicative form of Chlamydiaceae, cell division is reversibly arrested by the second binary fission in the presence of penicillin, resulting in four enlarged, non-infectious bodies $^{35}$. In free-living bacteria, the bactericidal effect of penicillin is owing to uncontrolled activity of PG hydrolases while PBP-conferred transpeptidase activity is blocked ${ }^{36}$. The genomes of Chlamydiaceae encode only one PG hydrolase, the cell division amidase AmiA. Uncontrolled AmiA activity does not lead to cell lysis in the isotonic niche and in the absence of a cell wall, making these 'minimal bacteria' an ideal tool to further analyse the action of beta-lactams beyond inhibition of PBP-catalysed transpeptidation and osmotic destabilization.

Previously, a role of amidases in generating a glycan-less wall polypeptide was proposed for Chlamydiaceae ${ }^{37}$. We have previously discussed a model for co-ordination of the lipid II pathway and cell division in Chlamydiaceae ${ }^{13,15}$ that implicates a central function of AmiA in sustaining an entire cycle of lipid II biosynthesis, processing and recycling (Fig. 6): MreB (in interaction with RodZ) functionally organizes lipid II biosynthesis at the septum and the synthesized building block is processed by a rudimentary cell wall biosynthesis/cell division machinery. Following translocation to the periplasm, AmiA and monofunctional transpeptidases PBP2 and FtsI (PBP3) process the cell wall-building block in a concerted way to facilitate the release of the bactoprenol carrier. In this step, the rudimentary PG-like product found by Liechti et al. ${ }^{18}$ in which the peptide side chains are cross-linked by peptide bonds might result. As our data show that AmiACp is also active against polymeric PG, we cannot rule out the possibility that the enzyme is involved in the turnover of the ring-like-shaped PG-like structure as well. The fate of the sugar units and turnover pathways for the cross-linked peptide side chains during the chlamydial developmental cycle 
are unknown. Moreover, recycling of bactoprenol-P remains unclear in the absence of pyrophoshorylases that have been described so far to dephosphorylate bactoprenol- $\mathrm{PP}^{38}$.

According to our model, chlamydial AmiA would play a crucial role in processing lipid II in the periplasm. Consistent with the lack of a Tat system in Chlamydiaceae, our data indicate that AmiA is secreted across the cytoplasmic membrane by the Sec system. Moreover, we showed that AmiA is a functionally conserved amidase in Chlamydiaceae and is capable of using the monomeric building block lipid II as a substrate. Surprisingly, chlamydial AmiA did not only cleave the peptide side chain from the sugar moieties, but also exhibited DD-CPase activity on lipid II and released the terminal D-Ala from the peptide side chain in vitro. In accordance with the observed sensitivity to penicillin, we identified an SxxK motif to mediate the novel DD-CPase activity in the bifunctional enzyme. Sporulation protein SpoIIP from B. subtilis has been shown to exhibit amidase and endopeptidase activity ${ }^{39}$, whereas Atl-like staphylococcal autolysins are bifunctional enzymes with amidase and $N$-acetylglucosaminidase activity ${ }^{40,41}$. To our knowledge, AmiACP is the first example of an enzyme possessing amidase and DD-CPase activity. Moreover, our study revealed that AmiA is a so far unknown target of penicillin in Chlamydiaceae, besides the PBPs PBP2, PBP3 and PBP6. It is not known whether homologous amidases from $E$. coli and other free-living pathogens have a dual activity such as chlamydial AmiA. Recruitment to the division site might be conferred by interaction with PBPs. For both PBP3 and PBP2 from C. trachomatis, interactions with the cell division protein FtsK have been shown in bacterial two-hybrid experiments ${ }^{42}$.

Many free-living bacteria have several low-molecular weight PBP DD-CPases, indicating an important but not completely understood physiological role for these peptidases. In E. coli the DD-CPase PBP5 is required for cell shape maintenance and proper division site placement, in particular, in mutants lacking other PG enzymes. Loss of PBP5 in a $\triangle a m i C$ mutant enhances chain formation and the helical morphology of FtsZ inhibitor SulA-induced filaments is dependent on PBP5 (ref. 20). E. coli PBP6 has been implicated in stabilizing the stationary phase PG and in the function of the cell division-specific transpeptidase PBP3 (ref. 20). Chlamydiaceae have a single PBP DD-CPase homologous to E. coli PBP6. PBP6, AmiA and Cpn0902 (NlpD) might together provide DD-CPase functions for chlamydial cell division.

Besides its role in cell division, the conserved lipid II pathway might contribute to sustaining long-term residence in infected cells and thus to pathogenicity. Induction of cytokines during chlamydial infections is not exclusively conferred by Toll-like receptor-dependent mechanisms or the adapter protein MyD88 signalling pathway ${ }^{1}$. Moreover, intracellular chlamydiae are recognized by human cytosolic pattern recognition receptors Nod1 and Nod2, which sense bacterial cell wall fragments to signal through a MyD88-independent pathway and mediate activation of the transcription factor $\mathrm{NF \kappa B}^{1}$. The amidase activity provided by AmiA could be involved in host response regulation (Fig. 6) comparable to eukaryotic PG recognition protein-L, which has amidase activity and may reduce host proinflammatory response to $\mathrm{PG}^{43}$. The concept of PG degradation as a common pathogenic strategy to subvert muropeptide recognition and host innate immunity has been described for other chronic human pathogens as well. Mycobacterium tuberculosis releases 'stealth' muropeptides catalysed by the resuscitation-promoting factors in concerted action with endopeptidase RipA or enzymes that modify pentapeptide side chains $^{43}$. In Helicobacter pylori, AmiA activity blocks Nod2 sensing as this receptor is incapable of recognizing released peptide side chains disconnected from an intact $\mathrm{N}$-acetylmuramic acid sugar unit ${ }^{43,44}$.

Further studies on the processing machinery of lipid II in Chlamydiaceae are needed for a better understanding of the chlamydial anomaly and the functions of amidases in cell division. Moreover, deeper insights into immune modulation, long-term residence in infected cells and chlamydial persistence in patients will be achieved.

\section{Methods}

Bacterial strains and growth conditions. E. coli JM83 carrying AmiA expression plasmids and E. coli ADE24 were maintained on Luria Bertani (LB) agar plates containing $100 \mu \mathrm{g} \mathrm{ml}^{-1}$ ampicillin and $30 \mu \mathrm{g} \mathrm{ml}^{-1}$ chloramphenicol, respectively.

Growth kinetics. E. coli JM83 harbouring expression vectors was grown in LB and induced with $200 \mathrm{ng} \mathrm{ml}^{-1}$ anhydrotetracycline (AHT) at an $\mathrm{OD}_{600}$ of 0.6 .

Construction of E. coli ADE24. E. coli MHD41 carries a markerless deletion in $a m i B^{6}$. We first deleted $a m i A$ by P1 transduction with JW2428 from the Keio E. coli mutant strain collection ${ }^{45}$, followed by the excision of the kanamycin resistance gene. Next, the amiC gene was deleted by P1 transduction with JW5449 from the Keio collection, followed by removal of the kanamycin resistance gene. The amiC gene was amplified by PCR from pBADAmiC ${ }^{6}$ using the oligonucleotides SacIamiCup and HindIIIamiCdw (Supplementary Table 1), which introduce SacI and HindIII restriction sites upstream and downstream, respectively, of amiC. The amiC gene was then cloned in plasmid pBAD33 and the resulting pBAD33-amiCEc was transformed into the triple-amidase mutant to obtain strain ADE24.

Cloning of amiA. The amiA gene from C. pneumoniae GiD was amplified by PCR using the primers listed in Supplementary Table 1 and cloned into pASK-IBA2 and pASK-IBA3 using the BsaI restriction site to generate an N-terminal OmpA-leader peptide fused, C-terminal Strep-tagged protein for periplasmic overproduction and a native signal peptide containing, C-terminal Strep-tagged protein for complementation assays, respectively.

Site-directed mutagenesis. H67, S96, H136, S140, S185 and E207 in AmiACp were changed to Ala using the QuikChange Lighting Site-Directed Mutagenesis Kit (Agilent Technologies, Germany). The respective sense and antisense primers listed in Supplementary Table 1 were used according to the manufacturer's instructions. Correct base changes were confirmed by sequencing.

In vivo complementation assay. E. coli $\mathrm{ADE} 24$ harbours an E. coli amiC encoding pBAD33 vector and shows an inducible chain-forming phenotype in the presence of glucose (Fig. 2; Supplementary Table 1). E. coli ADE24 was transformed with vector IBA3-amiACp allowing for expression of AmiACp including its native $\mathrm{N}$-terminal signal peptide upon induction by AHT. Cells were grown in the same medium used for overproduction of AmiACp containing $30 \mu \mathrm{g} \mathrm{ml}^{-1}$ chloramphenicol, $100 \mu \mathrm{g} \mathrm{ml}^{-1}$ ampicillin, $0.8 \%$ glucose and $200 \mathrm{ng} \mathrm{ml}^{-1}$ AHT at $32{ }^{\circ} \mathrm{C}$. Samples were taken at an $\mathrm{OD}_{600}$ of 0.5 and complementation was monitored by microscopy.

AmiA overproduction and purification. E. coli JM83 cells transformed with IBA2-amiACp were grown in no-salt LB in presence of $300 \mathrm{mM}$ sucrose at $30^{\circ} \mathrm{C}$ until they reached an $\mathrm{OD}_{600}$ of 0.6 and induced by the addition of $200 \mathrm{ng} \mathrm{ml}^{-1}$ AHT. After $3 \mathrm{~h}$ of incubation at $30^{\circ} \mathrm{C}$, cells were harvested and purification was performed using the manufacturer's (IBA, Germany) protocol for cleared lysates. OmpA cleavage was controlled by MS as described below.

AmiA activity assay. Remazol staining of E. coli W3110 PG sacculi and dyerelease assays were performed as described previously with slight modifications ${ }^{8}$. Stained sacculi $(20 \mu \mathrm{l})$ were incubated at $37^{\circ} \mathrm{C}$ for $16 \mathrm{~h}$ with $4 \mu \mathrm{M}$ AmiA in $200 \mu \mathrm{l}$ $50 \mathrm{mM}$ Hepes, $\mathrm{pH} 7.5,20 \%$ dimethyl sulphoxide. In vitro activity assays using lipid II as a substrate were carried out in a final volume of $40 \mu \mathrm{l}$ containing $4 \mu \mathrm{M}$ purified protein, $2 \mathrm{nmol}$ lipid II, $50 \mathrm{mM}$ Tris, pH 7.5 and 5\% dimethyl sulphoxide and incubated for $4 \mathrm{~h}$ at $37^{\circ} \mathrm{C}$. Reaction products were extracted with $40 \mu \mathrm{l}$ of $n$-butanol/pyridine acetate $(2: 1 \mathrm{v} / \mathrm{v}, \mathrm{pH} 4.2)$ and analysed by TLC and MS as described previously ${ }^{13}$ and below, respectively. For TLC, silica was used as the stationary phase, whereas the mobile phase consisted of chloroform-methanolwater-ammonia carbonate (88:48:10:1). Spots were visualized by phosphomolybdic acid (PMA) staining. For penicillin inhibition assays, the AmpR resistance marker in the AmiACp expression vector was exchanged with CamR resistance marker. 
MS. For reaction products $1 \mu \mathrm{l}$ of the sample was placed onto a ground steel matrix assisted laser desorption ionization time-of-flight target plate and allowed to dry at room temperature (RT). Each sample was then overlaid with $1 \mu \mathrm{l}$ of matrix (saturated solution of 6-Aza-2-thiothymine in $50 \%$ ethanol/20 mM diammonium citrate) and air dried at RT again. Spectra were recorded in the reflector negative mode on a biflex III mass spectrometer (Bruker Daltonik, Germany). For protein analysis, $2 \mu \mathrm{l}$ of the sample were mixed with $2 \mu \mathrm{l}$ of $2 \%$ trifluoroacetic acid solution. After adding $2 \mu \mathrm{l}$ of matrix solution (2,5-DHAP/DAC), this ternary mixture was pipetted up and down until crystallization started. The suspension $(1 \mu \mathrm{l})$ was spotted onto an AnchorChip 600 target and allowed to dry at RT. The spectra were recorded in the linear positive mode on a biflex III mass spectrometer.

\section{References}

1. McCoy, A. J. \& Maurelli, A. T. Building the invisible wall: updating the chlamydial peptidoglycan anomaly. Trends Microbiol. 14, 70-77 (2006).

2. Hafner, L. M. \& Timms, P. Persistent chlamydial infections: role in inflammation and challenges for vaccine development. Mucosal Immunol. Update 13, 4-7 (2005).

3. Typas, A., Banzhaf, M., Gross, C. A. \& Vollmer, W. From the regulation of peptidoglycan synthesis to bacterial growth and morphology. Nat. Rev. Microbiol. 10, 123-136 (2012).

4. Lutkenhaus, J., Pichoff, S. \& Du, S. Bacterial cytokinesis: From Z ring to divisome. Cytoskeleton (Hoboken) 69, 778-790 (2012).

5. Pinho, M. G., Kjos, M. \& Veening, J. W. How to get (a)round: mechanisms controlling growth and division of coccoid bacteria. Nat. Rev. Microbiol. 11, 601-614 (2013).

6. Heidrich, C. et al. Involvement of N-acetylmuramyl-L-alanine amidases in cell separation and antibiotic-induced autolysis of Escherichia coli. Mol. Microbiol. 41, 167-178 (2001)

7. Priyadarshini, R., de Pedro, M. A. \& Young, K. D. Role of peptidoglycan amidases in the development and morphology of the division septum in Escherichia coli. J. Bacteriol. 189, 5334-5347 (2007).

8. Uehara, T., Parzych, K. R., Dinh, T. \& Bernhardt, T. G. Daughter cell separation

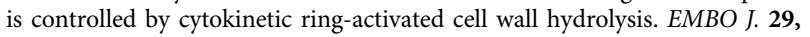
1412-1422 (2010).

9. Lupoli, T. J. et al. Studying a cell division amidase using defined peptidoglycan substrates. J. Am. Chem. Soc. 131, 18230-18231 (2009).

10. McCoy, A. J. et al. L,L-diaminopimelate aminotransferase, a trans-kingdom enzyme shared by Chlamydia and plants for synthesis of diaminopimelate/ lysine. Proc. Natl Acad. Sci. USA 103, 17909-17914 (2006).

11. Patin, D., Bostock, J., Blanot, D., Mengin-Lecreulx, D. \& Chopra, I. Functional and biochemical analysis of the Chlamydia trachomatis ligase MurE. J. Bacteriol. 191, 7430-7435 (2009).

12. De Benedetti, S. et al. Characterization of serine hydroxymethyltransferase GlyA as a potential source of D-alanine in Chlamydia pneumoniae. Front. Cell. Infect. Microbiol. 4, 19 (2014).

13. Henrichfreise, B. et al. Functional conservation of the lipid II biosynthesis pathway in the cell wall-less bacteria Chlamydia and Wolbachia: why is lipid II needed? Mol. Microbiol. 73, 913-923 (2009).

14. Patin, D., Bostock, J., Chopra, I., Mengin-Lecreulx, D. \& Blanot, D. Biochemical characterisation of the chlamydial MurF ligase, and possible sequence of the chlamydial peptidoglycan pentapeptide stem. Arch. Microbiol. 194, 505-512 (2012).

15. Gaballah, A., Kloeckner, A., Otten, C., Sahl, H. G. \& Henrichfreise, B. Functional analysis of the cytoskeleton protein MreB from Chlamydophila pneumoniae. PLoS ONE 6, e25129 (2011).

16. Pilhofer, M. et al. Discovery of chlamydial peptidoglycan reveals bacteria with murein sacculi but without FtsZ. Nat. Commun. 4, 2856 (2013).

17. Jacquier, N., Frandi, A., Pillonel, T., Viollier, P. \& Greub, G. Cell wall precursors are required to organize the chlamydial division septum. Nat. Commun. 5, 3578 (2014).

18. Liechti, G. W. et al. A new metabolic cell-wall labelling method reveals peptidoglycan in Chlamydia trachomatis. Nature 506, 507-510 (2014).

19. Joris, B. et al. The active-site-serine penicillin-recognizing enzymes as members of the Streptomyces R61 DD-peptidase family. Biochem. J. 250, 313-324 (1988)

20. Ghosh, A. S., Chowdhury, C. \& Nelson, D. E. Physiological functions of D-alanine carboxypeptidases in Escherichia coli. Trends Microbiol. 16, 309-317 (2008).

21. Arthur, M., Depardieu, F., Cabanié, L., Reynolds, P. \& Courvalin, P. Requirement of the VanY and VanX D,D-peptidases for glycopeptide resistance in enterococci. Mol. Microbiol. 30, 819-830 (1998).

22. Wright, G. D., Molinas, C., Arthur, M., Courvalin, P. \& Walsh, C. T. Characterization of vanY, a DD-carboxypeptidase from vancomycin-resistant Enterococcus faecium BM4147. Antimicrob. Agents. Chemother. 36, 1514-1518 (1992).

23. Hooper, N. M. Families of zinc metalloproteases. FEBS Lett. 354, 1-6 (1994).
24. Reynolds, P. E., Ambur, O. H., Casadewall, B. \& Courvalin, P. The VanY(D) DD-carboxypeptidase of Enterococcus faecium BM4339 is a penicillin-binding protein. Microbiology 147, 2571-2578 (2001).

25. Yang, D. C., Tan, K., Joachimiak, A. \& Bernhardt, T. G. A conformational switch controls cell wall-remodelling enzymes required for bacterial cell division. Mol. Microbiol. 85, 768-781 (2012).

26. Holm, L. \& Rosenström, P. Dali server: conservation mapping in 3D. Nucleic Acids Res. 38, W545-W549 (2010).

27. Hourdou, M. L. et al. Characterization of the sporulation-related gamma-Dglutamyl-(L)meso-diaminopimelic-acid-hydrolysing peptidase I of Bacillus sphaericus NCTC 9602 as a member of the metallo(zinc) carboxypeptidase A family. Modular design of the protein. Biochem. J. 292(Pt 2): 563-570 (1993).

28. Goffin, C. \& Ghuysen, J. M. Biochemistry and comparative genomics of SxxK superfamily acyltransferases offer a clue to the mycobacterial paradox: presence of penicillin-susceptible target proteins versus lack of efficiency of penicillin as therapeutic agent. Microbiol. Mol. Biol. Rev. 66, 702-738 (2002).

29. Vollmer, W., Blanot, D. \& de Pedro, M. A. Peptidoglycan structure and architecture. FEMS Microbiol. Rev. 32, 149-167 (2008).

30. Dilks, K., Rose, R. W., Hartmann, E. \& Pohlschröder, M. Prokaryotic utilization of the twin-arginine translocation pathway: a genomic survey. J. Bacteriol. 185, 1478-1483 (2003).

31. Bendtsen, J. D., Nielsen, H., Widdick, D., Palmer, T. \& Brunak, S. Prediction of twin-arginine signal peptides. BMC Bioinformatics 6, 167 (2005).

32. Petersen, T. N., Brunak, S., von Heijne, G. \& Nielsen, H. SignalP 4.0: discriminating signal peptides from transmembrane regions. Nat. Methods $\mathbf{8}$, 785-786 (2011).

33. Yang, D. C. et al. An ATP-binding cassette transporter-like complex governs cell-wall hydrolysis at the bacterial cytokinetic ring. Proc. Natl Acad. Sci. USA 108, E1052-E1060 (2011).

34. Frandi et al. FtsZ-independent septal recruitment and function of cell wall remodelling enzymes in chlamydial pathogens. Nat. Commun. 5:4200.

35. Skilton, R. J. et al. Penicillin induced persistence in Chlamydia trachomatis: high quality time lapse video analysis of the developmental cycle. PLoS ONE $\mathbf{4}$, e7723 (2009)

36. Tomasz, A. The mechanism of the irreversible antimicrobial effects of penicillins: how the beta-lactam antibiotics kill and lyse bacteria. Annu. Rev. Microbiol. 33, 113-137 (1979).

37. Ghuysen, J. M. \& Goffin, C. Lack of cell wall peptidoglycan versus penicillin sensitivity: new insights into the chlamydial anomaly. Antimicrob. Agents Chemother. 43, 2339-2344 (1999).

38. Tatar, L. D., Marolda, C. L., Polischuk, A. N., van Leeuwen, D. \& Valvano, M. A. An Escherichia coli undecaprenyl-pyrophosphate phosphatase implicated in undecaprenyl phosphate recycling. Microbiology 153, 2518-2529 (2007).

39. Morlot, C., Uehara, T., Marquis, K. A., Bernhardt, T. G. \& Rudner, D. Z. A highly coordinated cell wall degradation machine governs spore morphogenesis in Bacillus subtilis. Genes Dev. 24, 411-422 (2010).

40. Bourgeois, I. et al. Characterization of AtlL, a bifunctional autolysin of Staphylococcus lugdunensis with $\mathrm{N}$-acetylglucosaminidase and $\mathrm{N}$-acetylmuramoyl1-alanine amidase activities. FEMS Microbiol. Lett. 290, 105-113 (2009).

41. Oshida, T. et al. A Staphylococcus aureus autolysin that has an $\mathrm{N}$-acetylmuramoyl-L-alanine amidase domain and an endo-beta-Nacetylglucosaminidase domain: cloning, sequence analysis, and characterization. Proc. Natl Acad. Sci. USA 92, 285-289 (1995).

42. Ouellette, S. P., Karimova, G., Subtil, A. \& Ladant, D. Chlamydia co-opts the rod shape-determining proteins $\mathrm{MreB}$ and $\mathrm{Pbp} 2$ for cell division. Mol. Microbiol. 85, 164-178 (2012).

43. Humann, J. \& Lenz, L. L. Bacterial peptidoglycan degrading enzymes and their impact on host muropeptide detection. J. Innate Immun. 1, 88-97 (2009).

44. Girardin, S. E. et al. Peptidoglycan molecular requirements allowing detection by Nod1 and Nod2. J. Biol. Chem. 278, 41702-41708 (2003).

45. Baba, T. et al. Construction of Escherichia coli K-12 in-frame, single-gene knockout mutants: the Keio collection. Mol. Syst. Biol. 2, 2006.0008 (2006).

46. Juhasz, P. \& Costello, C. E. Matrix-assisted laser desorption ionization time-offlight mass spectrometry of underivatized and permethylated gangliosides. J. Am. Soc. Mass. Spectrom. 3, 785-796 (1992).

47. Hunter, S. et al. InterPro in 2011: new developments in the family and domain prediction database. Nucleic Acids Res. 40, D306-D312 (2012).

48. Krogh, A., Larsson, B., von Heijne, G. \& Sonnhammer, E. L. Predicting transmembrane protein topology with a hidden Markov model: application to complete genomes. J. Mol. Biol. 305, 567-580 (2001).

\section{Acknowledgements}

B.H. was supported by the European Union ('New Antimicrobials' project, people programme, FP7). H.B. and C.O. received a $\mathrm{PhD}$ fellowship from the Jürgen Manchot foundation. S.D.B. holds a fellowship from the NRW International Graduate Research School Biotech-Pharma. Support was received by the intramural funding scheme of the Medical Faculty of Bonn, BONFOR, and the Fonds der Chemischen Industrie. A.D. was supported by a long-term Fellowship by the European Molecular Biology Organization 
(EMBO). H.-G.S. and B.H. are members of the DFG Cluster of Excellence ImmunoSensation. We thank Patrick Viollier for communication of unpublished data.

\section{Author contributions}

B.H., A.K., C.O. and H.-G.S. designed research; experiments were performed by A.K., C.O., A.D., H.B., S.D.B., D.M., M.J. and K.M.; A.D. and W.V. developed analytical tools; C.O., A.K., B.H., H.-G.S., W.V., M.J., A.D., H.B., S.D.B., D.M. and K.M. analysed data; B.H., A.K., C.O., W.V. and H.B. wrote the paper.

\section{Additional information}

Supplementary Information accompanies this paper at http://www.nature.com/ naturecommunications

Competing financial interests: The authors declare no competing financial interests.
Reprints and permission information is available online at http://npg.nature.com/ reprintsandpermissions/

How to cite this article: Klöckner, A. et al. AmiA is a penicillin target enzyme with dual activity in the intracellular pathogen Chlamydia pneumoniae. Nat. Commun. 5:4201 doi: 10.1038/ncomms5201 (2014).

(c) (i) (2) This work is licensed under a Creative Commons Attribution(c) 1 BY NC SA NonCommercial-ShareAlike 4.0 International License. The images or other third party material in this article are included in the article's Creative Commons license, unless indicated otherwise in the credit line; if the material is not included under the Creative Commons license, users will need to obtain permission from the license holder to reproduce the material. To view a copy of this license, visit http:// creativecommons.org/licenses/by-nc-sa/4.0/ 